\title{
La cuestión rural en los programas de los partidos políticos en las elecciones de 2019
}

\section{The rural aproach in the programs of the political parties in the 2019 elections}

\author{
María Ángeles Abellán López \\ Universitat de València, Valencia, España \\ maria.a.abellan@uv.es \\ Gonzalo Pardo Beneyto \\ Universidad de Alicante, Alicante, España \\ gonzalo.pardo@ua.es
}

\begin{abstract}
Resumen: El presente artículo tiene como principal objetivo realizar un análisis de contenido de las propuestas electorales dirigidas al ámbito rural de los principales partidos que concurrieron a las elecciones legislativas de 2019. Para ello hemos utilizado el análisis de contenido y el software CAQDAS. Esta herramienta nos ha permitido clasificar las medidas en materialistas/postmaterialistas y productivistas/postproductivistas y determinar los principales términos utilizados. Entre las principales conclusiones del estudio podemos destacar que todos los partidos políticos incluyeron medidas desarrollistas y que la principal diferenciación se produjo entre bloques.
\end{abstract}

Palabras clave: Rural proofing, partidos políticos, agricultura, desarrollo rural, valores.

Abstract: The main objective of this article is to do a content analysis of the measures aimed at the rural sphere. In order to carry out it we have studied the electoral programs of the main political parties that attended the 2019 legislative elections. We have used the content analysis through a CAQDAS software. This tool has allowed us to classify the measures according to whether they were materialist/postmaterialist and productivist/postproductivist, as well as to determine the main terms used. Among the main ones of the study we can highlight that all the political parties included developmental measures and that the main differentiation occurred between blocks. values.

Key Words: Rural proofing, political parties, agriculture, rural development, 


\section{INTRODUCCIÓN}

Desde hace varias décadas existe preocupación sobre numerosos problemas del mundo rural y sus consecuencias para el conjunto del país. La falta de oportunidades vitales, el despoblamiento rural, el deterioro económico y la necesidad de infraestructuras han mermado la calidad de vida de la población rural. El fenómeno demográfico ha adquirido una enorme relevancia, y prueba del interés suscitado son los numerosos trabajos y estudios realizados en el último lustro, tanto en el ámbito académico (Reig, Goerlich y Cantarino, 2016; Franco, 2015; Camarero, 2009; Collantes, Pinilla y Sáez, 2010) como en el político (Comisión sobre Despoblación del Senado; Comisión de Despoblación de la FEMP; Alto Comisionado para el Reto Demográfico; Informe sobre el medio rural y su vertebración social y territorial del Consejo Económico y Social de España; Agenda 2030, entre otros) y en el de la sociedad civil (Red de Áreas Escasamente Pobladas del sur de Europa SSPA, plataformas territoriales reivindicativas y asociaciones diversas), con sus numerosos informes y estudios que evidencian la honda preocupación sobre esta cuestión.

Para la distinción entre lo rural y lo urbano suele recurrirse a umbrales demográficos como categorías clasificatorias. Así, el Instituto Nacional de Estadística (INE) toma como referencia el tamaño del hábitat, y se califica como rural los municipios inferiores a 10.000 habitantes. Sin embargo, para la Ley 45/2007, de Desarrollo Sostenible del Medio Rural (LDSMR), el municipio rural de pequeño tamaño es el que posee una población residente inferior a los 5.000 habitantes y está integrado en el medio rural. Según la Federación Española de Municipios y Provincias (FEMP), de los 8.125 municipios que tiene España, 4.995 tienen menos de mil habitantes. Por otro lado, el Alto Comisionado para el Reto Demográfico afirma que entre 2001 y 2017 el 61,7\% de los municipios españoles perdieron población. La Unión Europea considera territorios en riesgo de despoblación los que están por debajo de 12,5 habitantes por $\mathrm{km}^{2}$, que en España alcanza el $48 \%$ de los municipios. Lo cierto es que la pérdida de población se ha hecho extensiva no solo a los municipios inferiores a los 5.000 habitantes, sino también a un $70 \%$ de las cabeceras comarcales, entendidas como los núcleos funcionales de las áreas rurales, y al 63\% de las ciudades de entre 20.000 y 50.000 habitantes.

Este bloque de preocupaciones se ha canalizado recientemente hacia la agenda política doméstica y de manera más intensa se ha incorporado a los programas electorales de los partidos políticos en 2019, año con varias convocatorias de elecciones en los distintos niveles administrativos del Estado. 
La escasez de infraestructuras, la necesidad de diversificación económica, o la pérdida de peso de la agricultura como sector económico esencial, se aducen como factores condicionantes de la falta de incentivos para permanecer en el medio rural.

La sociedad rural ha experimentado un intenso proceso de diferenciación interno que la ha hecho mucho más compleja, lo que ha ofrecido una nueva estructura de oportunidades para la acción política. El reconocimiento de este diagnóstico ha generado un caldo de cultivo reivindicativo que eclosionó con la movilización ciudadana conocida como la revuelta de la "España vaciada" en 2019 y durante los primeros meses de 2020. El enfoque de la Estructura de Oportunidades Políticas (EOP) explica por qué se establecen conexiones y sinergias entre una determinada coyuntura política y la emergencia de actores colectivos (McAdam, 2002; Tarrow, 2012; Della Porta y Diani, 2011).

Los partidos políticos no son ajenos a los cambios del mundo rural y la evidencia empírica demuestra que se apoyan en las preferencias del electorado para definir sus alineamientos y captar el voto. Por esta razón, si en la opinión pública hay temas que son importantes para la ciudadanía, las fuerzas políticas se ven impelidas a hablar sobre ellos. Recientemente han comenzado a desarrollarse teorías sobre cómo las percepciones de los votantes dan forma a sus preferencias electorales, es decir, sobre el papel que tienen las percepciones de los problemas para determinar el voto. De este modo, mientras la teoría de ownership argumenta que los partidos suelen primar temas que interesan a sus votantes o aquellos temas donde tienen un eficaz dominio porque son de su "propiedad", la teoría de la convergencia sostiene que los partidos ganan a los votantes cuando demuestran que atienden los problemas que los electores consideran importantes, independientemente de la "propiedad" de tales problemas (Kaplan, Park y Ridout, 2006; Sides, 2006; Wagner y Meyer, 2014; Lefevere, Tresch y Walgrave, 2015). Por otro lado, este artículo considera esencial la contextualización del medio rural español en el marco de la Política Agraria Común (PAC) de la Unión Europea (UE) al que se refieren los partidos políticos en sus programas electorales, lo que viene a ser una replicación isomórfica.

El mundo agrario rural ha experimentado intensas transformaciones y los estudios rurales ofrecen diversos modelos y paradigmas para interpretar tales cambios. En este trabajo abordaremos la transición experimentada por el sistema agropecuario de la UE a través de una sucesión de modelos: productivismo, postproductivismo y neoproductivismo (Bowles, 1992, 1996; Marsden, 1992; Declaración de Cork, 1996, 2016; Bowler \& Ilvory, 1998; Evans, 1996, 2001; Hervieu, 1997; Lockwood, 1999; Evans, Morris y Winter, 2002; Armesto, 2005; OCDE, 2006, 2019; Wilson, 2007; Shortall, 2008; Marsden, 2012; Wilson y Burton, 2015). 
Asimismo, estableceremos vínculos teóricos con la dicotomía de valores materialista y postmaterialista (Inglehart, 1977), que crea un marco apropiado para explorar los aspectos agrorrurales en las propuestas de los partidos. Por ejemplo, el paso del productivismo al postproductivismo hace que lo rural sea percibido no solo como un locus de producción, sino como un espacio que aporta valores relacionados con la ecología, el paisaje, la justicia ambiental y el cambio climático, la alimentación saludable y la sostenibilidad, valores de interés y necesarios para una sociedad globalizada e interdependiente. En este contexto postproductivista es donde aparece el concepto de desarrollo rural, como se plasmó en la LDMRS. El desarrollo rural cuenta con una larga tradición en los estudios rurales, sin embargo, en este trabajo seguiremos la cronología de la PAC y de la $\operatorname{OCDE}(2006,2019)$, lo que implica que la conceptualización de lo rural como diferente de lo agrario hunde sus raíces en el cambio del viejo al nuevo paradigma (OCDE, 2006), es decir, el paso del productivismo al postproductivismo.

En esta investigación sostenemos que el productivismo se corresponde con valores de carácter materialista; el postproductivismo, con valores postmaterialistas, y el neoproductivismo apela a valores neomaterialitas. El neomaterialismo se considera un tipo de materialismo de nueva firma que más allá del valor per se de las cosas asigna un valor material incalculable a cosas que se daban por conseguidas y seguras. Por ejemplo, la crisis incrementó la inseguridad personal, laboral y económica, pero, a su vez, el envejecimiento y el despoblamiento rural amenazan la supervivencia de esas comunidades y el mantenimiento de ciertos servicios públicos.

Las consecuencias de la crisis económica de 2009 están en la raíz de las transformaciones rurales y de la importancia de valores materiales en las propuestas programáticas rurales como consecuencia de los efectos isomórficos de la PAC en el ejercicio 2014-2020, en el que la agricultura ha vuelto a ser el epicentro del desarrollo rural.

Con este andamiaje teórico conceptual hemos elaborado una taxonomía que establece un espectro político de cuatro categorías resultantes de combinar: por un lado, materialismo/postmaterialismo, y por otro, los modelos rurales-agropecuarios productivismo, postproductivismo y neoproductivismo que fundamentan el marco teórico.

La disposición de este trabajo es la siguiente: tras esta introducción, en primer lugar, se establece la metodología utilizada; en segundo lugar, se aborda el marco teórico; a continuación, en tercer lugar, se desarrolla la construcción de la tipología que enmarca este trabajo; y, en cuarto lugar, se analizan los resultados de esta investigación y finalmente se ofrecen las conclusiones. 


\section{METODOLOGÍA}

Para llevar a cabo este trabajo de investigación hemos utilizado el análisis de contenido para poder establecer inferencias entre los casos de estudio seleccionados. El propósito principal de esta metodología es la generación de una serie de indicadores que sirven para el análisis de los documentos propuestos (Corbetta, 2007). En consecuencia, este puede ser definido como "[...] una técnica de investigación destinada a formular, a partir de ciertos datos, inferencias reproducibles y válidas que puedan aplicarse a su contexto" (Krippendorf, 1990: 29).

Con el objetivo de mejorar el análisis interpretativo propuesto, hemos utilizado un CAQDAS ${ }^{1}$ que sirve para ayudar en el análisis e interpretación de los datos cualitativos. En concreto, nos ha permitido codificar y categorizar partes del texto, así como destacar el principal léxico utilizado en los documentos investigados. Dicha técnica computerizada nos ha permitido generar resultados cuantificables que nos ayudan a formar una imparcialidad objetiva (Cardoso, Queiroz, Zabdiele, Fernandes Ney, 2019).

En concreto, el software utilizado ha sido el WEBQDA, programa para analizar datos cualitativos basado en un entorno web que permite trabajar de forma colaborativa en tiempo real (Costa, 2016). Esta herramienta nos ha permitido analizar los principales programas electorales de las elecciones legislativas de abril y noviembre de 2019 para los cinco principales partidos de ámbito estatal presentes en el Parlamento español en la XIII legislatura.

En concreto, los partidos políticos elegidos por su relevancia a nivel del sistema de partidos han sido: Partido Socialista Obrero Español (PSOE), Partido Popular (PP), Ciudadanos (C's), Unidas Podemos (UP) y VOX.

Además, las convocatorias de 2019 permiten detectar cierta heterogeneidad en el sistema de partidos español de ámbito estatal, que se ha consolidado en un pluralismo moderado de estas fuerzas políticas como partidos efectivos (Sartori, 2005), o, en palabras de Morlino (2010), como multipartidismo heterogéneo y gobiernos de coalición.

Si bien es cierto que las convocatorias de 2019 están muy cercanas en el tiempo, las propuestas electorales rurales analizadas conjuntamente proporcionan una visión global que permite el estudio de los alineamientos partidistas en un entorno en el que parece que el sistema de partidos está en fase de estabilización.

${ }^{1}$ Con este acrónimo estamos haciendo referencia a software de análisis de datos cualitativos asistido por ordenador. 
Por otro lado, en las convocatorias electorales de 2015 y 2016 no aparecen elaboradas con cierto nivel de singularidad las propuestas rurales, lo que transmite la idea de que el interés por la cuestión rural es muy reciente.

Para realizar dicho análisis hemos seguido un plan de trabajo deductivo-cerrado (Costa \& Amado, 2019) que ha constado de las siguientes etapas:

1) En primer lugar, hemos definido una pregunta de investigación y un marco teórico y, a través de este, se han diseñado una serie de categorías.

2) Antes de empezar con el análisis hemos definido las unidades a tratar. Nos hemos centrado en las propuestas políticas relacionadas con la agricultura, la ganadería y la pesca, y con el desarrollo rural y sus principales problemáticas, como el reto demográfico, la economía y la falta de servicios.

3) Seguidamente, hemos codificado las propuestas según las categorías propuestas. Para ello hemos intentado que las medidas fueran la unidad de codificación principal. No obstante, ha habido varias medidas que tenían una relación directa con algunas de las categorías a través de su léxico o su amplitud, por lo que los fragmentos de estas han sido codificados de forma separada. Además, hemos elaborado una taxonomía en la que se ha combinado el par materialista/postmaterialista y productivista/postproductivista.

También se han generado nubes de palabras en las que se han escogido aquellas que tuviesen, per se, una significación propia. Se han eliminado palabras de menos de seis letras y aquellas que no aportasen valor como sustantivos genéricos, determinantes y adverbios, y, después, se han incluido las principales seis palabras.

Una vez hemos realizado esto, se han validado los datos y se han eliminado duplicidades en su ordenación.

4) Finalmente, se han trabajado los datos juntamente con las fuentes consultadas para la elaboración de una discusión y la comprobación de las hipótesis de trabajo.

Una vez hemos definido la metodología, es el momento de pasar a los referenciales teóricos que han marcado el análisis de la documentación y su codificación.

\section{REFERENCIALES TEÓRICOS}

\subsection{Modelos rurales agropecuarios en el marco de la Unión Europea}

Si bien los problemas rurales no son nuevos, lo cierto es que de un tiempo a esta parte ha cambiado la percepción social sobre el mundo rural. El foco de atención se 
dirige a un amplio abanico de fenómenos sociales y económicos del medio rural como el despoblamiento, la masculinización, el envejecimiento, el desempleo juvenil, la desigualdad o la dependencia. Además, la escasez de infraestructuras y la necesidad de diversificación económica se aducen como factores condicionantes de la falta de incentivos para permanecer en el medio rural. Este renovado interés por lo que ocurre en el medio rural se enfoca como un nuevo proceso de ruralización, diferente de la atención que se le dispensaba como un mundo aislado, distante y al que se identificaba de manera preferente con la actividad agraria y la cultura campesina (Moyano, 2005).

La sociedad rural ha experimentado un intenso proceso de diferenciación interno que la ha hecho mucho más compleja, lo que ha ofrecido una nueva estructura de oportunidades para la acción política. El reconocimiento de este diagnóstico ha generado un caldo de cultivo reivindicativo que eclosionó con la movilización ciudadana conocida como la revuelta de la "España vaciada" en 2019 y durante los primeros meses de 2020.

¿Por qué la cuestión rural interesa ahora a los partidos políticos y todos la incluyen en sus programas electorales de 2019?

El enfoque de la Estructura de Oportunidades Políticas (EOP) explica las conexiones y sinergias entre una determinada coyuntura política y la emergencia de actores colectivos (McAdam, 2002; Tarrow, 2012; Della Porta y Diani, 2011). Las movilizaciones son utilizadas por una ciudadanía que carece de acceso regular a las instituciones, que actúa en nombre de reivindicaciones nuevas o no atendidas, que está dispersa territorialmente, como es el caso de la ciudadanía rural, y que, además, cuestiona a sus élites representativas, que no han articulado eficazmente sus intereses territoriales.

Este trabajo considera que es necesario ofrecer una contextualización del mundo rural español a través de los condicionamientos isomórficos de la UE y sobre todo de la PAC. Este contexto nos va a proporcionar las claves sustantivas para interpretar las propuestas de los partidos políticos seleccionados en este trabajo.

Es importante precisar que bajo el rótulo de agricultura se incluye también las actividades de caza, pesca, silvicultura, cultivo de cosechas y cría de animales, de acuerdo con el Banco Mundial y la OCDE. Es incuestionable que la agricultura tiene un peso muy escaso en la economía española y que apenas representaba el 2,6\% del PIB en 2018. Un reciente estudio de FEDEA (2020) señala que en el periodo 1955-2018, el peso de la agricultura en el empleo nacional se ha desplomado desde el $46 \%$ hasta el $4 \%$ actual.

La economía rural de base agraria ha sufrido numerosos cambios derivados de la modernización económica global. Tales cambios han trazado una sucesión de modelos agropecuarios que son: el modelo productivista, el modelo postproductivista y el mo- 
delo neoproductivista (Bowles, 1992, 1996; Marsden, 1992; Bowles \& Ilvory, 1998; Evans, 1996, 2001; Hervieu, 1997; Lockwood, 1999; Evans, Morris y Winter, 2002; Armesto, 2005; Wilson, 2007; Shortall, 2008; Marsden, 2012; Wilson y Burton, 2015).

Junto a la evolución de estos modelos agropecuarios, también es necesario atender al cambio de paradigma rural propuesto por la $\mathrm{OCDE}$, que implica el tránsito del viejo paradigma al nuevo paradigma rural y su reciente adaptación 3.0 en la versión de 2019 (OCDE, 2006, 2019). El cruce de estos dos paradigmas y los tres modelos agropecuarios señalados más arriba nos va a permitir profundizar y captar las implicaciones de las propuestas programáticas de los partidos.

El modelo productivista ha sido hegemónico hasta los años 80 del siglo xx. Se caracteriza por la industrialización de la agricultura basada en una concepción tecnoeconómica fordista fundamentada en tres características: intensificación, concentración y especialización de la producción (Bowles, 1992). A grandes rasgos, y siguiendo a Lockwood, las señas de identidad del productivismo eran las siguientes: a) el peso que otorgaba a las ayudas y subvenciones públicas que garantizaban los precios; b) su fuerte dependencia de inputs de otros sectores económicos (fertilizantes, pesticidas, maquinaria), lo que incrementaba el consumo de energía y los desechos contaminantes; c) la sustitución de los factores productivos tierra y trabajo por el capital, lo que se tradujo en aumento del desempleo y descenso de la población rural; d) el papel subsidiario de la biodiversidad por el que se promovían nuevos cultivos, aplicando la biotecnología, y se dejaban extinguir variedades tradicionales en nombre de la eficiencia económica; y, e) la marcada diferenciación entre los roles del hombre y de la mujer en el ámbito agrícola. El productivismo comenzó a percibirse como enemigo de la biodiversidad y se definía por dos palabras: explotar y subsidiar (Lockwood, 1999).

En la década de los 90 apareció la expresión postproductivismo, que connotaba la idea de un cambio importante en las prioridades agrícolas que ya no se centraban en el incremento de la producción alimentaria (Bowles \& Ilvory, 1998; Bowles, 1996; Hervieu, 1997; Evans, 2001). Este cambio se caracterizaba por: a) la preferencia por la calidad y no por la cantidad, b) la expansión y diversificación de las actividades, c) el énfasis en la sostenibilidad a través de políticas agroambientales y d) el interés en la regulación de los impactos medioambientales en la agricultura (Evans, 2001). El modelo postproductivista se alineaba con las características del nuevo paradigma rural de la OCDE (2006), en el que se destacaba una nueva ruralidad centrada en valores y prácticas que enfatizaban el desarrollo territorial endógeno, la diversificación de la economía rural, el cambio climático y la importancia de sectores no agrícolas. De esta manera, pasaron a primer plano aspectos como el valor paisajístico, la sostenibilidad, la justicia 
ambiental, la agricultura verde, el ocio, el turismo, el patrimonio, la artesanía, el folklore, la resforestación y una demanda de alimentos ecológicos saludables y de calidad. Es decir, el mundo rural deja de ser percibido como una superficie de producción para ser enfocado como un espacio que puede aportar valores de interés para la sociedad global urbana, en el que la calidad se erige en el concepto clave (Armesto, 2005). Desde el punto de vista axiológico, enlazaría con el paso de valores materialistas a postmaterialistas (Inglehart, 1977).

Sin embargo, la crisis económica mundial de 2009 supuso un punto de inflexión que originó un cambio en el paradigma de desarrollo rural y adivinó un nuevo modelo que comenzó a conocerse como neoproductivismo fuertemente asociado al neoliberalismo (Marsden, 2014). La crisis económica y los problemas de adaptación al cambio climático pasaron a primer plano. El neoproductivismo recupera la importancia del sector agrícola como instrumento para el desarrollo rural, para crear empleo y para luchar contra el cambio climático. Por esta razón, diversos autores afirman que el neoproductivismo es una versión más sofisticada del productivismo (Evans, Morris y Winter, 2002), que representa una involución, un paso atrás en el fomento del desarrollo rural (Shortall, 2013), la eliminación del apoyo a actividades no agrícolas y un retorno a los subsidios.

Wilson y Burton (2015) criticaron las insuficiencias del término neoproductivismo, pero, a su vez, reconocieron que el concepto facilita a los investigadores afinar las conceptualizaciones de los complejos cambios espaciales, temporales y estructurales del mundo rural agropecuario actual, puesto que la contraposición modelo productivista/ postproductivista se revela inadecuada para dar cuenta de las transformaciones recientes.

En la Tabla 1 se ofrece una síntesis de los tres modelos agropecuarios de la UE que se han tratado en las líneas precedentes.

Respecto a los cambios de paradigmas propuestos por la OCDE, que como sabemos tienen un enfoque y alcance globalizadores, ha actualizado el modelo del nuevo paradigma de 2006 para reconvertirlo en la Política Rural 3.0.

De esta manera, bajo el telón de fondo de los modelos de desarrollo agropecuarios que hemos visto, vamos a superponer el nuevo paradigma que propone la OCDE, que reconoce tanto los objetivos económicos como los sociales y medioambientales. El Rural 3.0 es un enfoque que pretende centrarse en las personas y no en los sectores económicos, y se caracteriza particularmente por una división de zonas rurales según la densidad, la adopción de tecnologías digitales, la adaptación al cambio demográfico, el envejecimiento de la población rural, la transición ecológica y el acceso a servicios públicos de calidad. 
TABLA 1

Paradigmas de la política rural-agraria de la UE

\begin{tabular}{|c|c|c|c|}
\hline $\begin{array}{c}\text { Paradigmas } \\
\text { Características }\end{array}$ & $\begin{array}{l}\text { Productivismo } \\
\text { hasta } 1980\end{array}$ & $\begin{array}{c}\text { Postproductivismo } \\
1980-2010\end{array}$ & $\begin{array}{l}\text { Neoproductivismo } \\
2010 \text {-actualidad }\end{array}$ \\
\hline $\begin{array}{l}\text { Carácter del } \\
\text { área rural }\end{array}$ & $\begin{array}{l}\text { Espacio productivo } \\
\text { agrícola }\end{array}$ & $\begin{array}{l}\text { Espacio de consumo, } \\
\text { mercantilización del } \\
\text { campo }\end{array}$ & $\begin{array}{l}\text { Espacio vulnerable a la } \\
\text { incertidumbre económica y } \\
\text { ecológica actual }\end{array}$ \\
\hline Objetivos & $\begin{array}{l}\text { Producción } \\
\text { agrícola y } \\
\text { estabilización de la } \\
\text { población agrícola } \\
\text { (estabilidad de } \\
\text { precios e ingresos) }\end{array}$ & $\begin{array}{l}\text { Agricultura } \\
\text { multifuncional y } \\
\text { apoyo a actores no } \\
\text { agrícolas en áreas } \\
\text { rurales para } \\
\text { impactos más } \\
\text { amplios }\end{array}$ & $\begin{array}{l}\text { Agricultura es la herramienta } \\
\text { para mitigar el cambio } \\
\text { climático, favorecer el } \\
\text { crecimiento verde como } \\
\text { respuesta a la crisis económica } \\
\text { de } 2009-2010 \text {, apoyo a la } \\
\text { agricultura sostenible }\end{array}$ \\
\hline $\begin{array}{l}\text { Política } \\
\text { económica }\end{array}$ & $\begin{array}{l}\text { Proteccionismo y } \\
\text { regulación }\end{array}$ & Desregulación & Vuelta a política reguladora \\
\hline $\begin{array}{l}\text { Herramientas } \\
\text { y conceptos }\end{array}$ & $\begin{array}{l}\text { Enfoque sectorial } \\
\text { Subsidios }\end{array}$ & $\begin{array}{l}\text { Mix de herramientas } \\
\text { sectoriales y } \\
\text { estructurales }\end{array}$ & $\begin{array}{l}\text { Modelo de desarrollo con } \\
\text { énfasis en pagos ambientales } \\
\text { Subsidios }\end{array}$ \\
\hline $\begin{array}{l}\text { Herramientas } \\
\text { para el } \\
\text { desarrollo } \\
\text { rural }\end{array}$ & $\begin{array}{l}\text { Dispersas en otras } \\
\text { políticas } \\
\text { económicas de la } \\
\text { UE }\end{array}$ & $\begin{array}{l}\text { Inversiones } \\
\text { Concentración de } \\
\text { herramientas en la } \\
\text { política de desarrollo } \\
\text { rural PAC, pilar II }\end{array}$ & $\begin{array}{l}\text { Regreso a la política } \\
\text { estructural de la UE; } \\
\text { fragmentación; desarrollo } \\
\text { territorial integrando } \\
\text { relaciones urbanas-rurales; } \\
\text { soluciones a la crisis } \\
\text { económica }\end{array}$ \\
\hline Actores clave & $\begin{array}{l}\text { CEE/UE } \\
\text { Estados miembros } \\
\text { Agricultores }\end{array}$ & $\begin{array}{l}\text { UE, Estados } \\
\text { miembros, } \\
\text { agricultores, ONG, } \\
\text { municipios, } \\
\text { empresarios no } \\
\text { agrícolas }\end{array}$ & $\begin{array}{l}\text { UE y agricultores, empresas } \\
\text { no agrícolas en un número } \\
\text { limitado }\end{array}$ \\
\hline $\begin{array}{l}\text { Métodos de } \\
\text { cultivo }\end{array}$ & $\begin{array}{l}\text { Intensificación y } \\
\text { explotación } \\
\text { del medio ambiente }\end{array}$ & $\begin{array}{l}\text { Extensificación y } \\
\text { protección ambiental }\end{array}$ & Intensificación sostenible \\
\hline $\begin{array}{l}\text { Enfoque de } \\
\text { desarrollo }\end{array}$ & $\begin{array}{l}\text { Cantidad de } \\
\text { producción } \\
\text { Agricultura como } \\
\text { sinónimo de lo } \\
\text { rural }\end{array}$ & $\begin{array}{l}\text { Desarrollo } \\
\text { sostenible, desarrollo } \\
\text { endógeno, } \\
\text { diversificación, } \\
\text { calidad }\end{array}$ & $\begin{array}{l}\text { Crecimiento verde, agricultura } \\
\text { sostenible con desarrollo } \\
\text { neoendógeno, mantenimiento } \\
\text { de tierras agrícolas y } \\
\text { forestales. }\end{array}$ \\
\hline
\end{tabular}

Fuente: Adaptación propia a partir de Ilbery (1998), Marsden (2014), OCDE (2006, 2019), Wilson (2001), Woods (2011), Wilson \& Burton (2015) y Pelucha \& Kveton (2017). 
En la Tabla 2 se visualizan las características de los paradigmas de la OCDE.

TABLA 2

Paradigmas de la política rural-agraria de la OCDE

\begin{tabular}{|c|c|c|c|}
\hline & $\begin{array}{c}\text { Viejo } \\
\text { paradigma } \\
\text { Hasta } 2005\end{array}$ & $\begin{array}{c}\text { Nuevo } \\
\text { paradigma } \\
2005-2019\end{array}$ & $\begin{array}{c}\text { Rural 3.0: a people-centred } \\
\text { approach } 2019 \\
\text { (nueva adaptación } \\
\text { paradigma 2006) }\end{array}$ \\
\hline Objetivos & $\begin{array}{l}\text { Enfoque de } \\
\text { igualación o } \\
\text { derecho, centrado } \\
\text { en el ingreso } \\
\text { agrícola, la } \\
\text { competitividad } \\
\text { agrícola }\end{array}$ & $\begin{array}{l}\text { Competitividad de las } \\
\text { zonas rurales, } \\
\text { valorización de los } \\
\text { activos locales, } \\
\text { explotación de los } \\
\text { recursos no utilizados }\end{array}$ & $\begin{array}{l}\text { Objetivos sociales, políticos, } \\
\text { económicos y ambientales; } \\
\text { reconocimiento de diversidad } \\
\text { de desafíos y oportunidades } \\
\text { en las distintas áreas rurales }\end{array}$ \\
\hline Meta clave & $\begin{array}{l}\text { Sectorial } \\
\text { (unisectorial) }\end{array}$ & $\begin{array}{l}\text { Diversos sectores de } \\
\text { las economías rurales } \\
\text { (turismo rural, } \\
\text { manufactura, industria } \\
\text { de las TIC, etc.) }\end{array}$ & $\begin{array}{l}\text { Enfoque centrado en las } \\
\text { personas }\end{array}$ \\
\hline $\begin{array}{l}\text { Herramientas } \\
\text { principales }\end{array}$ & Subsidios & Inversiones & $\begin{array}{l}\text { Tecnologías digitales, } \\
\text { inversiones, herramientas de } \\
\text { transición climatológica y } \\
\text { demográfica }\end{array}$ \\
\hline Actores clave & $\begin{array}{l}\text { Gobiernos } \\
\text { nacionales, } \\
\text { agricultores }\end{array}$ & $\begin{array}{l}\text { Gobierno multinivel, } \\
\text { partes interesadas } \\
\text { locales (público, } \\
\text { privado, ONG), } \\
\text { gobernanza rural }\end{array}$ & $\begin{array}{l}\text { Público } \\
\text { Privado } \\
\text { Sociedad civil } \\
\text { Tercer sector }\end{array}$ \\
\hline
\end{tabular}

Fuente: OCDE (2016, 2019).

En consecuencia, existe una pluralidad de intereses y de valores representados por diferentes actores del mundo rural, en el que se entrecruzan diversos modelos agropecuarios y políticas de desarrollo rural.

Todo ello transmite un vivo dinamismo a la sociedad rural y ofrece una nueva estructura de oportunidades a los diferentes grupos sociales cuyas demandas heterogéneas han sido recogidas por los partidos políticos en sus programas electorales. 


\subsection{Cambio y valores en los partidos políticos}

Inglehart (1977) plantea una teoría de cambio cultural esencial para comprender la modernidad en la que se produce el paso de valores materialistas a los valores postmaterialistas, que se inicia a partir de la II Guerra Mundial. Los valores materialistas son los valores tradicionales relacionados con la seguridad física y económica, la estabilidad, la autoridad, que son los que predominan en sociedades preindustriales e industriales. Inglehart observa la aparición de nuevas tendencias que favorecen los valores postmaterialistas y que se relacionan con la autoexpresión, la realización personal, la calidad de vida y el medioambiente. La pirámide de Maslow sirvió para fundamentar el cambio cultural, y lo que en principio tenía una aplicación individual la trasladó al orden social. El cambio de valores se consideró bajo dos hipótesis: la hipótesis de la escasez y la hipótesis de la socialización. La primera de las hipótesis es una adaptación de la jerarquía de necesidades de Maslow: los individuos buscan satisfacer sus necesidades y cuando cubren aquellas de seguridad física y económica, buscan el desarrollo de valores postmateriales. La hipótesis de la socialización argumenta que el ambiente en que los ciudadanos se desarrollan condiciona su estructura y prelación de valores. Los primeros años de socialización definen las pautas de actuación de los individuos, por lo que estas prioridades persisten a lo largo de la vida. La complementariedad de las dos hipótesis ofrece una explicación del cambio de valores en las sociedades occidentales.

Desde el punto de vista social, la estabilidad y el crecimiento económico, la seguridad, el Estado de bienestar, la tecnología y la expansión educativa son factores determinantes de estos cambios hacia los valores postmaterialistas. De esta forma, aparece un interés político por temas como la identidad, la pertenencia, los estilos de vida y elementos específicos.

Diferentes investigaciones pusieron de manifiesto que los grupos e individuos y grupos muestran diferentes prioridades con respecto a sus valores, cuya observación es relevante para comprender la realidad en la sociedad contemporánea, como es la persistencia de valores tradicionales, aunque exista prosperidad económica. El par materialis$\mathrm{mo} /$ postmaterialismo ha sido, incluso, considerado como un cleavage en el alineamiento de los partidos políticos. Así, una visión diferente es la sostenida por Della Porta y Diani (2011) cuando afirman que quizás lo correcto no sea interpretar el postmaterialismo como una nueva línea divisoria, sino justamente lo inverso. Cuando por razones históricas y específicas surge una nueva fractura puede que esta se organice alrededor de la divisoria materialismo/postmaterialismo. Lo cierto es que la aparición de valores postmaterialistas genera un realineamiento de los viejos partidos y, en general, posiciona a todos los partidos existentes. 


\section{ENCUADRAMIENTO DE UNA TIPOLOGÍA}

Los partidos políticos investigados han incorporado a sus programas electorales la cuestión rural. En el referencial teórico se ha expuesto la sucesión de modelos rurales agropecuarios en el marco de la PAC y de las políticas públicas de la UE, donde se incardinan las propuestas de las formaciones políticas españolas. Tales modelos hay que considerarlos como tipos puros en sentido weberiano, lo que significa que son categorías con valor estrictamente metodológico. $\mathrm{O}$, dicho de otro modo: estas categorías recogen los rasgos más significativos de un determinado fenómeno, puesto que no existen en estado puro, pero que nos sirven para sintetizar y entender la realidad.

Para la elaboración de las categorías hemos combinado los paradigmas productivismo/postproductivismo y los valores materialismo/postmaterialismo. El modelo neoproductivista (y su correlato neomaterialismo) que hemos presentado a efectos expositivos no se ha categorizado por varias razones.

En primer lugar, no existe unanimidad sobre su conceptualización y alcance en los investigadores especialistas. En segundo lugar, los debates académicos se han centrado principalmente en el cambio agrícola y apenas ha recibido atención el desarrollo rural. En tercer lugar, falta investigación sobre el neoproductivismo y su literatura es muy reciente y, entendemos, en proceso de elaboración y reelaboración constante.

Las propuestas electorales se han sistematizado a partir de la combinación de dos criterios. Por un lado, los valores materialistas y postmaterialistas y, por el otro, los paradigmas productivista y postproductivista. En consecuencia, la relación entre los datos (programas electorales) y el marco teórico en que se fundamenta este trabajo nos permite establecer categorías (codificación teórica).

La combinación resultante ofrece una tipología como se muestra en la Tabla 3, que nos permitirá desarrollar el análisis. Las cuatro categorías analíticas son conservacionismo, tradicionalismo, desarrollismo y evolucionismo.

TABLA 3

Categorización de las propuestas electorales sobre el desarrollo rural

\begin{tabular}{lll}
\hline \multicolumn{1}{c}{ Paradigma/Valores } & \multicolumn{1}{c}{ Materialismo } & \multicolumn{1}{c}{ Postmaterialismo } \\
\hline Productivismo & Conservacionismo & Tradicionalismo \\
Postproductivismo & Desarrollismo & Evolucionismo \\
\hline
\end{tabular}

Fuente: Elaboración propia.

Estas categorías permiten identificar las propuestas electorales rurales de las fuerzas políticas consideradas que describimos a continuación. 
Conservacionismo. Esta categoría aglutina valores materialistas con elementos del paradigma productivista. Se enfatizan los aspectos de seguridad física y económica; se reafirma la defensa de sectores y estructuras tradicionales y, además, se otorga un peso importante a las subvenciones como estabilizadoras de los precios. Mantiene un enfoque top-down, lo que produce rigidez estructural y una política proteccionista e intervencionista. Hay un claro predominio sectorial de la agricultura sobre cualquier otra actividad basada en la producción de alimentos y primando la cantidad sobre la calidad. Asimismo, se equipara la agricultura como sinónimo de lo rural. La superficie rural se define por su capacidad de producción agraria y aspira a estabilizar la población agrícola. Las políticas de desarrollo rural no agrario quedan subsumidas en otras políticas públicas de manera subsidiaria. Por otro lado, se comparte una visión común de la comunidad rural que no atiende o muestra interés por las especificidades territoriales o la diversidad de factores contextuales que están presentes. Los ítems que se tomarán en cuenta para su operacionalización son los siguientes:

1) La agricultura, la ganadería, la silvicultura, la cría de ganado y la pesca son actividades fundamentales en el territorio.

2) Lo agrícola es sinónimo de rural.

3) El resto de usos del campo están subordinados a los anteriores.

4) El modelo conservacionista es de carácter productivista, sectorial y proteccionista, centrado en la seguridad.

5) El enfoque político se orienta, fundamentalmente, a la seguridad (propiedad privada) y a la producción agrícola, ganadera y pesquera (cantidad de producción).

Desarrollismo. Esta categoría resulta de combinar valores materialistas con el paradigma postproductiva. Si bien prioriza elementos de seguridad física y económica, también impulsa la diversificación productiva y el cambio tecnocrático. La tendencia es primar la agricultura de calidad en detrimento de la cantidad. Las subvenciones son importantes por su capacidad estabilizadora, pero sin desdeñar otras herramientas e inversiones. El contexto desarrollista facilita la emergencia de nuevas estructuras de oportunidades que involucra a los nuevos y viejos actores; se amplía la base social de la gobernanza rural orientada al desarrollo endógeno. Existe, pues, una mayor diferenciación dentro y fuera del sector agrario y mayor diversificación de actividades y profesiones, lo que reduce el protagonismo de las élites agrarias tradicionales. En el modelo desarrollista comienza a detectarse cierta sensibilización hacia prácticas agrícolas más respetuosas por el medio ambiente. Los ítems que se tomarán en cuenta para su operacionalización son los siguientes: 
1) La agricultura, la ganadería y la pesca no son las únicas vías para el desarrollo económico rural.

2) Se fomenta un apoyo explícito al turismo rural, a la transformación agrícola, ganadera y pesquera a través de procesos industrializados.

3) Se genera otro tipo de negocios en todos aquellos bienes que se pueden mercantilizar relacionados con la gestión del paisaje, la cultura, el patrimonio natural y cultural, la construcción de segundas residencias, el deporte, etc.

4) Preferencia del uso de técnicas respetuosas con el medio ambiente en detrimento de las técnicas nocivas, cumplimiento de legalidad y energías renovables.

5) Las medidas políticas adoptadas se orientan a la mejora del nivel de vida de la ciudadanía rural, ampliación de servicios públicos y nuevas tecnologías.

Tradicionalismo. Esta categoría combina elementos del modelo productivista con valores postmaterialistas. Su formulación comporta la defensa de los valores tradicionales de la comunidad rural, de rearme moralizante de la cultura y el rechazo a los procesos estandarizados de la globalización. En consecuencia, se mantiene una visión conservadora, orgánica y unitaria de lo rural acompañada de bajos niveles de confianza y/o escepticismo hacia las instituciones, aunque con dependencia de las ayudas y subvenciones públicas. La política de desarrollo rural no puede escindirse de la agrícola, y configuran una unidad. Desde el punto de vista del productivismo, la agricultura es la actividad principal caracterizada por la intensificación, la concentración y la especialización de la producción (Bowles, 1992). Los actores agrícolas son los que tienen peso en el desarrollo rural en detrimento de los agentes no agrícolas. El modelo de desarrollo económico no favorece la diversificación y existe una mayor rigidez estructural del sistema rural con enfoque top-down. Los ítems que se tomarán en cuenta para su operacionalización son los siguientes:

1) Los modos de vida rurales tradicionales son un bien que hay que salvaguardar, por lo que actividades como la caza, la tauromaquia o las tradiciones ancestrales (religiosas o celebraciones para tener buenas cosechas) han de ser preservadas.

2) La globalización es un fenómeno que produce rechazo, especialmente la cultural y desconfianza hacia las instituciones.

3) Fuerte resistencia a los cambios socioculturales.

4) Mantenimiento de un imaginario rural y de la división social del trabajo agrícola.

5) Enfoque top-down, primacía de los actores agrícolas tradicionales y de las estructuras clásicas. 
Evolucionismo. Se trata de una categoría que hibrida valores postmaterialista con un paradigma postproductivista. Se prima el desarrollo rural como una vía independiente de la agricultura, la cual ha de ser verde y ecológica. La polivalencia del postproductivismo integra otros productos rurales como la artesanía, el turismo, el ocio y recreación, la reforestación y el patrimonio natural y cultural, que añaden nuevos usos y requerimientos al mundo rural. La ruralidad aporta valores y prácticas sociales muy valiosas para una sociedad global cada vez más urbana. Aparecen nuevos valores relacionados con el cambio climático, la sostenibilidad, la gestión del paisaje, la seguridad alimentaria, la alimentación saludable, la justicia ambiental y no eluden el debate sobre la modificación genética de alimentos, el especismo y el bienestar de los animales. Para esta categoría existe cierta diferenciación de los actores del medio rural que utilizan y aprovechan los mismos espacios y territorios pero que no necesariamente comparten un sistema común de valores, lo que abre el abanico axiológico. Además, apela a la gobernanza rural con enfoque bottom-up, atentos a las especificidades regionales y a las posibilidades de nuevas estructuras de oportunidades para la acción política. Los ítems que se tomarán en cuenta para su operacionalización son los siguientes:

1) Redefinición de la ruralidad basada en la calidad de vida, accesibilidad y ampliación de servicios públicos y lucha contra el cambio climático.

2) El desarrollo rural y la agricultura son dos vías complementarias y beneficiosas para la economía circular, el reciclaje, la diversificación, etc.

3) El hilo discursivo integra debates como gobernanza rural, justicia ambiental, bienestar de los animales, igualdad de género rural, etc.

4) Lo rural como parte del continuum urbano rural.

5) Impulso para implementar políticas públicas de participación y empoderamiento ciudadano.

A partir de esta cuádruple categorización hemos formulado las siguientes hipótesis de investigación ${ }^{2}$.

$\mathrm{H}_{1}$. Los partidos ubicados a la izquierda tendrán un mayor número de propuestas desarrollistas y evolucionistas.

2 Para la ubicación como de derecha o izquierda se ha tomado el Barómetro del CIS de septiembre de 2019, Estudio 3261, pregunta 28, que clasifica a los partidos políticos entre 1 y 10 , en el que 1 es izquierda y 10, derecha. Los resultados, tomando el valor medio, son los siguientes: PSOE, 4,1; PP, 7,9; C's, 7,1; UP, 2,$3 ;$ y VOX, 9,4. 
$\mathrm{H}_{2}$. Los partidos políticos situados a la derecha del espectro político tendrán un mayor número de propuestas conservacionistas y tradicionalistas.

El siguiente apartado se dedica al análisis de los resultados de las propuestas de los partidos políticos para las elecciones legislativas de abril y noviembre de 2019, que serán interpretadas a la luz de las categorías expresadas.

\section{RESULTADOS}

\subsection{Partido Socialista Obrero Español}

En cuanto al PSOE, la formación de centroizquierda tiene un sesgo manifiesto hacia las medidas desarrollistas complementadas con las evolucionistas. Entre abril y noviembre, los socialistas llevaron a cabo un viraje hacia postulados más materialistas, perdiendo parte de las medidas evolucionistas del mes de abril y adoptando otras de cariz conservacionista.

TABLA 4

Codificación de las medidas programáticas del PSOE

\begin{tabular}{lcccc}
\hline & Conservacionismo & Desarrollismo & Tradicionalismo & Evolucionismo \\
\hline Abril & 1 & 17 & 0 & 9 \\
Noviembre & 4 & 14 & 0 & 5 \\
\hline
\end{tabular}

Fuente: elaboración propia.

El PSOE cuenta con propuestas mayoritariamente desarrollistas; le siguen las evolucionistas y pocas conservacionistas. Estas últimas se refieren al reto demográfico, a infraestructuras y la ubicación de unidades militares en zonas despobladas. Entre las iniciativas socialistas, además del apoyo a la agricultura y ganadería extensiva, la silvicultura y la reforma de la PAC, destacan la diversificación y los usos de los espacios rurales, proponiendo el turismo rural y la biodiversidad como generadoras de riqueza, la multifuncionalidad de instituciones y agencias públicas (caso de Correos), emprendedurismo y autoempleo para la juventud, la digitalización y la Reforma del Plan Forestal Nacional y Estrategia de Desarrollo Rural. Respecto a las propuestas evolucionistas, se destacan en el PSOE las relacionadas con la salud oceánica, recogida de basura y plásticos en el mar, el corredor para cetáceos, la incorpora- 
ción de la biodiversidad para que compute en la contabilidad nacional y el teletrabajo. En definitiva, en las propuestas socialistas existen reiteraciones en diferentes medidas, sobre todo en el programa de abril, que pretenden abarcar un amplio campo semántico pero que conceptualmente son poco operativas. Carece de propuestas en la categoría tradicionalista.

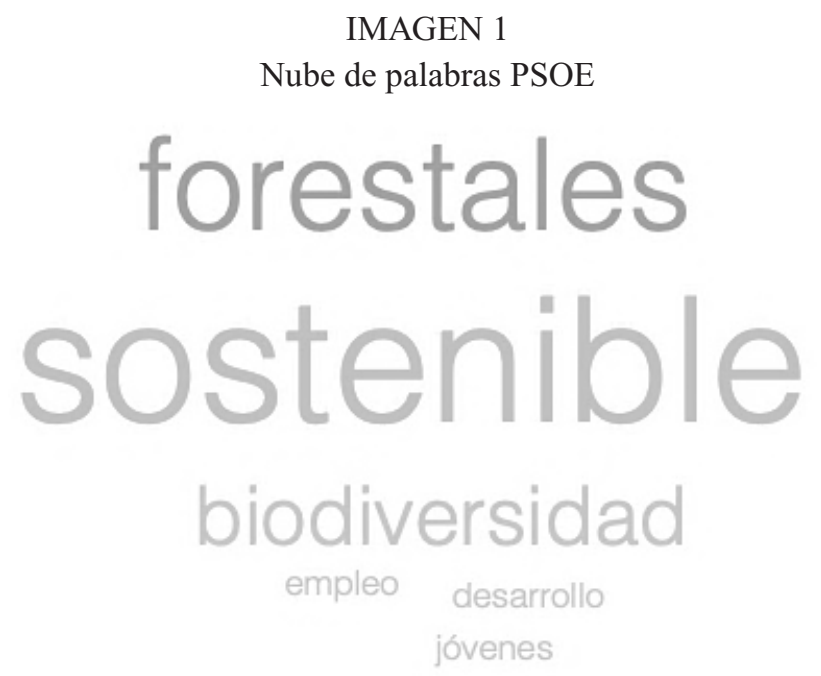

Fuente: elaboración propia.

Para el PSOE la sostenibilidad (11) es el término más importante y que subyace en todo el programa electoral. Para este partido la sostenibilidad es crecimiento, y por eso tiene ese protagonismo en el programa electoral. Los términos forestal (11) y biodiversidad (9) están presentes como elementos de riqueza y de bienestar personal. Otros términos como empleo (8), desarrollo (8) y jóvenes (8) se retroalimentan mutuamente, pues la juventud es un segmento de su interés.

En síntesis, en el PSOE encontramos un partido desarrollista y evolucionista y que se corresponde con el modelo postproductivista.

\subsection{Partido Popular}

El Partido Popular aplicó una racionalización en su programa electoral de noviembre de 2019 respecto a la versión de abril para las elecciones generales. 
TABLA 5

Codificación de las medidas programáticas del PP

\begin{tabular}{lcccc}
\hline & Conservacionismo & Desarrollismo & Tradicionalismo & Evolucionismo \\
\hline Abril & 9 & 23 & 3 & 7 \\
Noviembre & 4 & 6 & 3 & 2 \\
\hline
\end{tabular}

Fuente: elaboración propia.

E1 Partido Popular también es una fuerza política con vocación de gobierno y un partido "propietario" (ownership) del tema rural, con propuestas de calado y específicas que evidencian un conocimiento experto del mundo rural.

El PP tiene propuestas en todas las categorías en diversos grados, con clara hegemonía desarrollista. En la categoría conservacionista encontramos iniciativas que se corresponden con el modelo productivista. Así, por ejemplo, citamos las relacionadas con el despliegue de la Guardia Civil y la coordinación de las Policías locales por el territorio rural, la nueva negociación de la PAC como máxima prioridad, el plan de gestión de las crisis agrarias o la estabilización de rentas y la protección específica de cítricos, productos agrícolas y ganaderos. Respecto a las propuestas desarrollistas, son las más numerosas y abarcan temas como la agenda digital, una diferenciación fiscal para el medio rural, emprendedurismo y modernización agrarios y pesqueros, gestión forestal, turismo rural, inclusión de la perspectiva rural en la legislación y planes relacionados con el desarrollo rural, con los secanos tradicionales, con el cambio climático, Agrorenove, con el agua y regadíos y la fusión de diferentes servicios (veterinarios y sanidad). En el programa de noviembre, además, incluyó que el envejecimiento y la despoblación se conciban como factores en la ponderación para la reforma del sistema de financiación autonómica.

En cuanto a las medidas tradicionalistas, los populares establecen el Plan Nacional de Tauromaquia (PENTAURO) para que extienda el conocimiento y la difusión del arte taurino y potencie el conjunto de actividades alrededor de esta importante tradición; planes cinegéticos, apoyo a licencias de caza y pesca interautonómicas y énfasis en el papel de las diputaciones para el desarrollo rural como proveedoras de servicios públicos esenciales. Finalmente, las propuestas evolucionistas de los populares recogen la creación de clusters de conocimiento y experiencia agroalimentaria $\mathrm{I}+\mathrm{D}+\mathrm{i}$, el enfoque de género y el maltrato de la mujer, la economía circular en la agricultura, el cambio climático y la nueva gobernanza del agua. En resumen, la PAC, la transformación digital y el reto demográfico son elementos de peso en su discurso electoral con el apoyo de medidas económicas, fiscales y financieras en las diferentes políticas públicas rurales. 


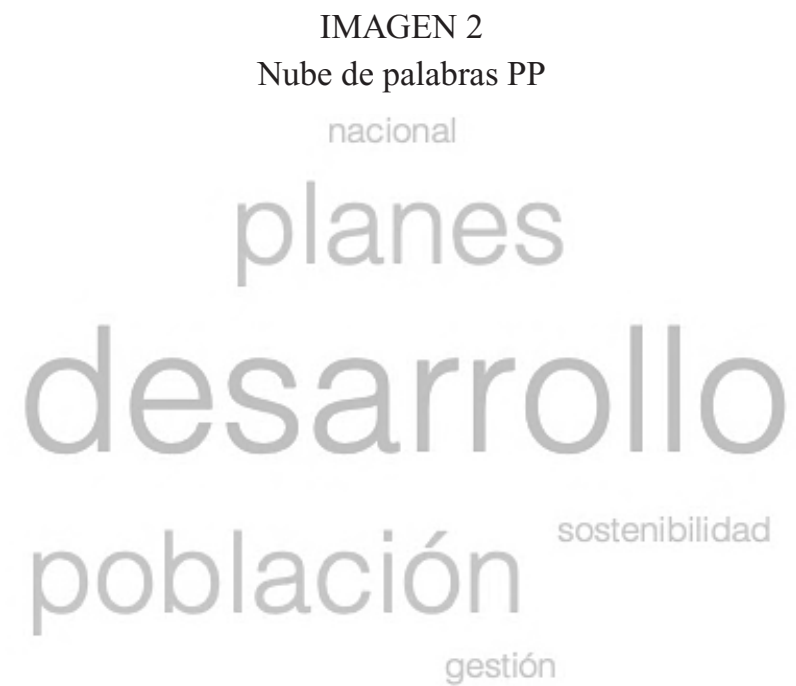

Fuente: elaboración propia.

Las palabras que subyacen dejan entrever el principal objetivo del Partido Popular, que es el desarrollo (14) de las zonas rurales. La palabra planes (11) nos indica el arraigo que tiene la formación conservadora en las zonas rurales y su acción de gobierno. No es extraño que dicha formación tenga un mayor conocimiento de las necesidades de dichas zonas y la articulación en programas político-administrativos coherentes que traten de mejorar las condiciones de vida materiales de su población (11). Alrededor de estos conceptos encontramos el medio ambiente rural (8), el papel de la idea de nación y sus valores propios (8) y las necesidades de la gestión de estas problemáticas desde una perspectiva política (8).

Por tanto, puede afirmarse que el PP es un partido desarrollista y a caballo entre el modelo postproductivista y neoproductivista.

\subsection{Ciudadanos}

En cuanto a las medidas propuestas por el partido naranja en su programa electoral podemos comprobar que ha habido pequeños realineamientos entre la convocatoria electoral de abril y la de noviembre. Sus medidas son prácticamente las mismas; no obstante, el alcance de alguna de ellas se ha visto modulado, cambiando de valores postmaterialistas a valores materialistas. 
TABLA 6

Codificación de las medidas programáticas de Ciudadanos

\begin{tabular}{lcccc}
\hline & Conservacionismo & Desarrollismo & Tradicionalismo & Evolucionismo \\
\hline Abril & 2 & 9 & 2 & 3 \\
Noviembre & 2 & 10 & 1 & 3 \\
Fuente: elaboración propia. & & & &
\end{tabular}

En Ciudadanos encontramos un programa electoral bastante parco sobre la cuestión rural. C's tiene propuestas en las cuatro categorías, con predominio de las de carácter desarrollistas. Las conservacionistas se refieren a reducciones impositivas en donaciones y sucesiones agrícolas. Respecto a las medidas desarrollistas, destacamos el emprendimiento rural, el pacto contra la despoblación, la fiscalidad diferenciada en municipios amenazados por el despoblamiento, prevención de incendios forestales, la brecha digital, el turismo rural, la PAC y profesionalización y tecnificación del sector agroalimentario, la contribución desde el sector agrario al Plan Integrado de Energía y Clima y el Plan Renove para maquinaria agrícola y forestal. Las medidas tradicionalistas de C's se orientan a la defensa y caza como actividades tradicionales en municipios rurales y la elaboración de inventario del patrimonio cultural en el mundo rural. Ciudadanos también cuenta con propuestas evolucionistas como son los planes de reforestación para la preservación de la biodiversidad, lucha contra el cambio climático, planes de conservación de las especies protegidas y de gestión de especies invasoras.

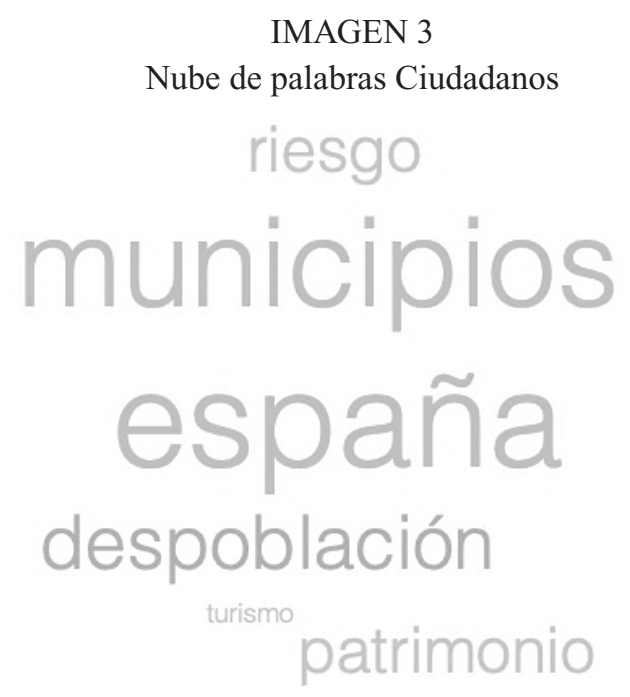

Fuente: elaboración propia. 
En cuanto a las palabras más utilizadas en estas medidas, debemos hacer referencia al uso de España (16) como un término unificador y generador de igualdad entre los distintos territorios del país. Esta idea queda reforzada en la retórica utilizada por Ciudadanos a través del uso de "municipio" como unidad básica al que se hace referencia en las medidas propuestas (15) y que se unen a la concepción de municipios viables y poblados. Por eso no es de extrañar que la palabra despoblación (12) esté presente, y también riesgo (11), en el que están inmersas las áreas rurales por el reto demográfico. Para C's el medio rural posee potencial para generar riqueza a través del patrimonio (11) y el turismo (9).

En definitiva, en el programa electoral de Ciudadanos escasean las referencias al desarrollo rural per se y, en todo caso, ofrece medidas de carácter fragmentario. En consecuencia, C's se puede caracterizar como un partido desarrollista con predominio de valores materialistas y acorde a un modelo neoproductivista.

\subsection{Unidas Podemos}

El programa de Unidas Podemos es el que más ideas evolucionistas recoge, aunque son superadas por las desarrollistas. Lo que caracteriza a UP es su mirada con perspectiva de justicia territorial, ambiental y de género para reducir las desigualdades.

\section{TABLA 7}

Codificación de las medidas programáticas de Unidas Podemos

\begin{tabular}{lcccc}
\hline & Conservacionismo & Desarrollismo & Tradicionalismo & Evolucionismo \\
\hline Abril & 3 & 14 & 0 & 12 \\
Noviembre & 3 & 14 & 0 & 12 \\
\hline
\end{tabular}

Fuente: elaboración propia.

Como se aprecia en la Tabla 7, Unidas Podemos cuenta con propuestas en tres categorías: conservacionista, desarrollista y evolucionista. No tiene ninguna propuesta tradicionalista. UP es una de las fuerzas políticas que más propuestas rurales abarca en su programa electoral y con cierto nivel de concreción y profundidad, tanto en la versión programática de abril como de noviembre de 2019.

Entre las medidas conservacionistas, se destaca la atención de la Guardia Civil, la protección a pequeñas empresas agropecuarias. Las principales propuestas desarrollistas engloban a la PAC y la Política Pesquera Común; los incendios forestales, la protec- 
ción a nuevos sectores de gran aporte social y ambiental (frutos secos, azafrán), diversos planes para el sector agropecuario (Plan Pymes 5.0, Plan Cooperación 5.0, Plan StartUp 5.0), juventud y bancos de tierras. Respecto a las medidas de carácter evolucionista, aglutina un abanico de iniciativas de distinta índole como: un enfoque prevencionista de incendios forestales durante el invierno, la transparencia, PAC como lanzadera de la transición ecológica, el pastoralismo y las vías pecuarias para conservar la biodiversidad y luchar contra el cambio climático, un registro de incidencias sobre contaminación y microplásticos, la protección de los derechos de los animales, la creación de una ley de bienestar animal, la eliminación de ayudas y subvenciones a la tauromaquia y prohibición de espectáculos de maltrato animal, un Plan Estratégico del Patrimonio Natural y la Biodiversidad (2020-2027), una oferta cultural descentralizada, la creación de instituciones para frenar el inframunicipalismo, la gestión democrática de servicios comunes y la convocatoria electoral para representantes de agricultura y ganadería.

\section{IMAGEN 4}

Nube de palabras Unidas Podemos
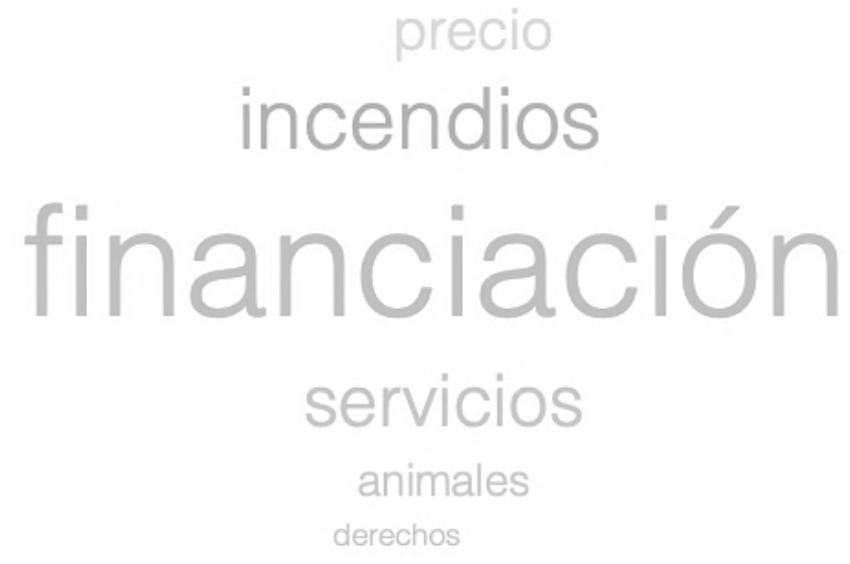

Fuente: elaboración propia.

Las medidas de Unidas Podemos están enmarcadas en un contexto evolucionista que las hacen distintas al resto de los partidos estudiados, lo que queda patente en palabras utilizadas en estas medidas como incendios (15), animales (12) y derechos (11). Hay que destacar que la formación morada utiliza como término más repetido el de financiación (20). Esto se explica por el papel del sector público y la necesidad de reforzar sus dotaciones en servicios esenciales y en la planificación de la actividad económica. Esta idea queda reforzada por el uso del término servicio (14) o el valor que trans- 
mite "precio", y que se engarza con la idea de que sin elementos reguladores del mercado de productos procedentes del sector primario difícilmente estos podrán tener una vida material plena.

En definitiva, Unidas Podemos es un partido a caballo entre el evolucionismo y el desarrollismo, pero cabe precisar que su evolucionismo marca el carácter de sus propuestas desarrollistas.

\subsection{VOX}

La Tabla 8 contiene la codificación de las medidas de VOX y se aprecia que es la misma en ambos programas, abril y noviembre de 2019, puesto que utilizó el mismo documento para ambas convocatorias. De todo lo señalado hasta ahora podemos afirmar que de los cinco programas analizados el de VOX es el que menos propuestas electorales rurales contiene, y las que hay carecen de concreción.

TABLA 8

Codificación de las medidas programáticas de VOX

\begin{tabular}{lllll}
\hline & Conservacionismo & Desarrollismo & Tradicionalismo & Evolucionismo \\
\hline Abril & 2 & 3 & 4 & 0 \\
Noviembre & 2 & 3 & 4 & 0 \\
\hline
\end{tabular}

Fuente: elaboración propia.

VOX está presente en el conservacionismo, desarrollismo y tradicionalismo. No tiene propuestas evolucionistas. Entre las propuestas conservacionistas encontramos la iniciativa de la revisión de la Ley del Suelo (que se aplica a todos los ámbitos, sean urbanos o rurales) y la rebaja a los impuestos de combustible, fertilizantes, herbicidas, plásticos. Entre las propuestas desarrollistas destacan la dotación de incentivos para profesionales agrarios y cursos de formación, la expansión de internet, la renovación del parque de maquinaria y la reducción de desigualdades entre el medio rural y urbano para evitar el despoblamiento. En el tradicionalismo es donde destaca VOX, categoría donde incluye propuestas como el fomento del arraigo a la tierra, las manifestaciones folclóricas y tradiciones de España y de sus pueblos dentro de la hispanidad; la ley de protección de la tauromaquia, como patrimonio cultural español; la caza, como actividad necesaria y tradicional del mundo rural, licencia única de caza a nivel nacional y eliminación del sistema de licencias autonómicas e interautonómicas, y la revisión del 
modelo de aplicación de la PAC de forma que las ayudas lleguen en mayor medida a quienes gestionan directamente las explotaciones agrícolas y viven principalmente de ello.

\section{IMAGEN 1}

Nube de palabras VOX

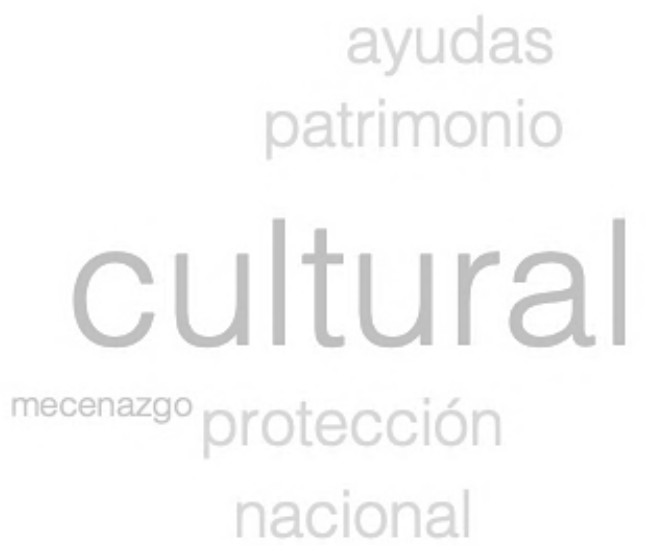

Fuente: elaboración propia.

En cuanto a la nube de palabras, esta pone de manifiesto la importancia que se le da al valor cultural (10) tradicional del campo que entronca con una idea nacional (4) representada por los valores rurales que han de ser protegidos (4) y fomentados a través de la ayuda pública (4) y las aportaciones privadas a través del mecenazgo (2).

El sesgo cultural tradicionalista de VOX condiciona las medidas conservacionistas y desarrollistas.

\section{CONCLUSIONES}

El presente artículo ha ofrecido un esfuerzo interpretativo para analizar las propuestas electorales rurales de los partidos políticos. La investigación desarrollada nos permite responder a las hipótesis que hemos planteado a lo largo del trabajo. Entre las principales conclusiones se destaca que el tema rural está presente en los programas electorales, lo que no significa que incluyan la perspectiva rural.

Los partidos políticos estudiados ofrecen propuestas de valores materiales y postmateriales en proporciones variables. Encontramos que las propuestas desarrollistas son 
comunes a todas las formaciones políticas estudiadas. Esto entendemos que se produce por el carácter transformador propio de la política y que aboca a los representantes políticos a ofrecer propuestas de desarrollo a sus potenciales votantes.

Las diferencias entre cada uno de los partidos estudiados se fundamentan en la composición del resto de propuestas. Tanto PSOE como Unidas Podemos no tienen ninguna medida tradicionalista en sus programas; no obstante, sí incluyen medidas conservacionistas relacionadas con la estabilidad de precios, el comercio y la seguridad. La diferencia entre ambas fuerzas políticas la encontramos en el lenguaje utilizado y la interrelación con el resto de las propuestas. Mientras que el PSOE tiene como objetivo fundamental mejorar la eficiencia productiva del mundo rural y subordina las propuestas evolucionistas a este hecho - aun utilizando la palabra sostenible en múltiples medidas-, Unidas Podemos integra el evolucionismo postmaterialista y postproductivista en el proceso productivo buscando un cambio social más profundo que los socialistas. Por eso, podemos afirmar que se cumple la hipótesis 1.

En cuanto al bloque de la derecha, el caso de VOX es el más radical y apela a valores de tipo postmaterial para construir un relato, en el que lo rural es un elemento diferenciador de la cultura española. Por ello, se postula para defender actividades como la tauromaquia y la caza. También incluyen medidas desarrollistas para remarcar la necesidad de que exista igualdad y unidad entre todos los españoles.

Tanto PP como C's combinan propuestas en todas las categorías y sus diferencias estriban en el control del tema estudiado en este artículo. El Partido Popular — sobre todo en abril- elaboró un documento con muchas medidas diversas. La razón principal es que se utilizó no solo para las elecciones generales, sino también para las elecciones autonómicas y locales. Por este hecho, el número de arenas políticas se multiplicaron y obligaron a la formación conservadora a desplegar una estrategia en la que se atrajesen al máximo número de votantes del ámbito rural. En noviembre, dichas propuestas se simplificaron.

La diferencia entre PP y C's también reside en el grado de especialización de las medidas estudiadas. Ciudadanos hizo del desarrollismo su principal seña de identidad, aunque trató de incluir propuestas de sus principales competidores para atraer a más votantes. Por esto cuenta con medidas conservacionistas y tradicionalistas en su programa electoral.

El Partido Popular no se queda atrás en la defensa de valores conservacionistas y tradicionalistas e incluye una defensa también hacia la cinegética y la cultura taurina. Asimismo, y desde un punto de vista conservacionista, está totalmente a favor de la defensa de los sectores agrícolas tradicionales y de garantizar la seguridad en todas sus vertientes. PP y C's incluyeron en menor grado propuestas evolucionistas. 
Con base en todo lo anterior, podemos afirmar que, aunque bien es cierto que las propuestas conservacionistas y tradicionalistas tienen un sentido simbólico fundamental, la hipótesis 1 no se confirma puesto que el PP y C's son desarrollistas y VOX, tradicionalista.

Para finalizar y quedarnos con una visión de conjunto, todos los partidos desarrollan estrategias para maximizar su presencia en el espectro político con propuestas isomórficas. En realidad, las diferencias obedecen a alineamientos según bloques ideológicos que han funcionado en las elecciones de 2019. En el bloque de izquierda, PSOE y UP apuestan por propuestas desarrollistas y evolucionistas de manera mayoritaria. En cuanto al bloque de derecha, PP, C's y VOX han singularizado sus propuestas en función de sus expectativas. PP y C's son partidos desarrollistas y VOX es tradicionalista.

\section{BIBLIOGRAFÍA}

Armesto López, Xosé A. (2005). "Notas teóricas en torno al concepto de postproductivismo agrario". Investigaciones Geográficas, 36: 137-156.

Bowles, I.R. (1992). "The industrialisation of agriculture". En Bowler, I.R. (ed.). The geography of agriculture in developed market economics (6-31). Nueva York: Longman Scientific and Technical.

- (1996). "Agricultural land use and the post-productivist transition". En López Ontiveros, A. y Molinero Hernando, F. (ed.). La investigación hispano-británica reciente en geografia rural: del campo tradicional a la transición postproductivista (179187). Asociación de Geógrafos Españoles, Murcia.

Camarero, L. (coord.). (2009). La población rural de España. De los desequilibrios a la sostenibilidad social. Colección Estudios Sociales, núm. 27. Fundación La Caixa.

Cardoso, C.S., de Queiroz, D., Zabdiele, M., Fernandes, R., Ney, F.R. (2019). "Contributions and Limits to the Use of Softwares to Support Content Analysis Clara". En Costa, A.P., Paulo, L., Moreira, A. (eds.). Computer Supported Qualitative Research (12-21). Cham: Springer.

Collantes, F., Pinilla, V., Sáez, L. A., Silvestre, J. (2010). El impacto demográfico de la inmigración en la España rural despoblada, Madrid: Real Instituto Elcano.

Comisión de las Comunidades Europeas (1996). The Cork Declaration. A Living Countryside, Cork: Comisión de las Comunidades Europeas.

Corbetta, P. (2007). Metodología y técnicas de investigación en sociología, Madrid: McGraw-Hill. 
Costa, A. P. (2016). “Cloud Computing em Investigação Qualitativa: Investigação Colaborativa através do software webQDA". Fronteiras, 5(2): 153-161. https://doi.org/10.21664/2238-8869.2016v5i2.p153-161.

Costa, A. P., y Amado, J. (2019). Análisis de contenido soportado por Software, Aveiro: Ludomedia.

De la Fuente, A., y Ruiz, P. (2020). Estudios sobre la Economía Española, 2020/08. Series largas de VAB y empleo regional por sectores, 1955-2018. Disponible en http://documentos.fedea.net/pubs/eee/eee2020-08.pdf (abril 2020).

Della Porta D. y Diani M. (2011). Los Movimientos Sociales. Madrid: Editorial Complutense. CIS.

Evans, N. (2001). "Reflexiones en torno al modelo productivista de la agricultura y la ganadería". En García Pascual, F. El mundo rural en la era de la globalización: incertidumbres y potencialidades (45-64). Lleida: Asociación de Geógrafos Españoles.

Evans, N., Morris, C., \& Winter, M. (2002). "Conceptualizing agriculture: a critique of post-productivism as the new orthodoxy". Progress in Human Geography, 26(3): 313-332. https://doi.org/10.1191/0309132502ph372ra

Federación Española de Municipios y Provincias (2017). Documento de trabajo de la Comisión de Despoblación de la FEMP.

Fernández-Albertos, José (2018). Antisistema. Desigualdad económica y precariado político, Madrid: La Catarata.

Franco, F. (2015). "Mundo, medio y territorio rural". Fundación Encuentro. Informe España 2015.

Halfacree, K. (1999). "A new space or spatial effacement? Alternative futures for the post-productivist countryside”. En Walford, N., Everitt, J. and Napton, D. (eds). Reshaping the countryside: perceptions and processes of rural change (67-76), Wallingford: CAB International.

Hervieu, B. (1997). Los campos del futuro, Madrid: Ministerio de Agricultura, Pesca y Alimentación.

Ilbery, B. (ed.) (1998). The Geography of Rural Change, Londres: Londman.

Ilbery, B. y Bowler, R. (1998). "From agricultural productivism to post-productivism". En Ilbery, B. (ed.). The Geography of Rural Change (57-84), Londres: Londman.

Inglehart, R. (1977). The Silent Revolution: Changing Values and Politica Styles Among Western Publics. Princeton: Princeton University Press.

Jollivet, M. (dir.) (1997). Vers un rural postindustriel. Rural et environnement dans huit pays européens, París: L'Harmattan. 
Kaplan, N., Park, D.K. y Ridout, T.N. (2006). "Dialogue in American Political Campaigns? An Examination of Issue Convergence in Candidate Television Advertising". American Journal of Political Science, 50: 724-736. https://doi. org/10.1111/j.1540-5907.2006.00212.x

Krippendorf, K. (1990). Metodologia de Analisis de Contenido, Barcelona: Paidós.

Lockwood, J.A. (1999). "Agriculture and biodiversity: finding our place in this world". Agriculture and Human Values, 16: 365-379.

Lowe, P., Murdoch J., Marsden, T., Munton, R., Flynn, A. (1993). "Regulating the new rural spaces: the uneven development of land". Journal of Rural Studies, 9(3): 205222.

Markus W. \& Meyer, T. (2014). Which Issues do Parties Emphasise? Salience Strategies and Party Organisation in Multiparty Systems. West European Politics, 37(5): 10191045.

Marsden, T.K. (1992). "Exploring a rural Sociology for the Fordist Transition". Sociologia Ruralis. Journal of the European Society for Rural Sociology. Vol. XXXII: 209230. https://doi.org/10.1111/j.1467-9523.1992.tb00929.x

- (1999). "Rural futures: the consumption countryside and its regulations". Sociologia Ruralis, 39: 501-520.

- (2012). "Food systems under pressure: regulatory instabilities and the challenge of sustainable development" (291-312). En Spaargaren G., Oosterveer, P., y Loeber, A. (eds.). Food practices in transition: changing food consumption, retail and production in the age of reflexive modernity, Nueva York: Routledge.

Maye, D., Enticott, G., y Naylor, R. (2020). "Theories of Change in Rural Policy Evaluation”. Sociologia Ruralis, 60: 198-221. https://doi.org/10.1111/soru.12269

McAdam, D. (2002). "Movimientos iniciadores y derivados: procesos de difusión en los ciclos de protesta". En Traugott, M., Protesta social. Barcelona: Hacer Editorial.

Morlino, Leonardo (2010). Introducción a la investigación comparada, Madrid: Alianza.

OCDE (2006). New Paradigme. Paris

- (2019). Rural 3.0: People Centred Rural Policy, Policy Highlights. París.

Pelucha, M. \& Kveton, V. (2017). "The role of EU rural development policy in the neo-productivist agricultural paradigm”. Regional Studies, 51(12): 1860-1870. doi: 10.1080/00343404.2017.1282608

Reig, E., Goerlich, F., y Cantarino, I. (2016). Delimitación de áreas rurales y urbanas a nivel local: demografia, coberturas y accesibilidad. Madrid: Fundación BBVA.

Sartori, Giovanni (2005). Partidos y sistemas de partidos, Madrid: Alianza. 
Sides, J. (2006). “The Origins of Campaign Agendas". British Journal of Political Science, 36(3): 407-436. https://doi.org/10.1017/S0007123406000226

Tarrow S.G. (2012). El poder en movimiento. Los movimientos sociales, la acción colectiva y la política. Madrid: Alianza.

Wilson, G. (2001). "From Productivism to Post-Productivism...and Back again? Exploring the (Un)changed Natural and Mental Landscapes of European Agriculture". Transactions of the Institute of British Geographers, 26(1): 77-102.

Wilson, G. \& Burton, R. (2015). "Neo-productivist agriculture: Spatio-temporal versus structuralist perspectives". Journal of Rural Studies, 38: 52-64.

Woods, M. (2011). Rural geography: processes, responses and experiences in rural restructuring, Londres: Sage. 